\title{
Emotion regulation, brain behavioural systems, and sensory sensitivity in sociocultural attitudes towards appearance in adolescents
}

\author{
Sohrab Amiri' ${ }^{1}$ Amir Ghasemi Navab² \\ 'Behavioral Sciences Research Center, Lifestyle Institute, Baqiyatallah University of Medical Sciences, Tehran, Iran \\ ${ }^{2}$ Allameh Tabatabaei University, Tehran, Iran
}

Neuropsychiatria i Neuropsychologia 2019; 14, 1-2: 32-38

\author{
Address for correspondence: \\ Sohrab Amiri, PhD \\ Behavioral Sciences Research Center \\ Lifestyle Institute \\ Baqiyatallah University of Medical Sciences \\ Tehran, Iran \\ e-mail: rsr.amiri.s@bmsu.ac.ir
}

\begin{abstract}
This study investigates the usefulness of the emotion regulation (ER) brain behavioural systems and sensory sensitivity to better understand sociocultural attitudes towards appearance. Specifically, associations between positive and negative ER, and incremental validity of brain behavioural systems and highly sensitive person (HSP) as predictors of the attitudes towards appearance are examined. Data were obtained in a sample of healthy adolescents $(N=331)$. Results of a series of multiple regression analyses showed that ER provided a significant prediction of each of the five attitudes towards appearance variables. Dimensions of brain behavioural systems including behavioural activation system (BAS), behavioural inhibition system (BIS), fight-flight-freeze system (FFFS) showed multiple associations with facets from the attitudes towards appearance. BAS and FFFS were found to be the positive predictors and BIS was a negative predictor. HSP was found to be a positive predictor of attitudes towards appearance. The results expand the understanding of the attitudes towards appearance and indicate how domains of ER, personality, and sensory processing could explain the attitude of adolescents towards their appearance.
\end{abstract}

Key words: emotion regulation, reinforcement sensitivity, sensory processing, attitudes towards appearance.

\section{Introduction}

Adolescents encounter situations that may evoke body image concerns (Dittmar et al. 2007). About 30-50\% of girls and boys have body image concerns (Thompson 2001; O'Dea and Yager 2006). Such concerns have adverse psychological consequences (Cash et al. 2004) such as depression and anxiety (Cash et al. 2004; Kostanski and Gullone 1998). Body image dissatisfaction can cause distress and have an impact on quality of life, social relationships, and academic functioning (Cash and Fleming 2002). Only some adolescences show psychological problems due to body image dissatisfaction (Cash 2002). It seems that body image concerns during adolescence may be transient experiences. Such differences are probably due to individual differences in risk and protective factors. One important factor in this regard, which has received little attention, is ER.
Emotion regulation (ER) is a process through which emotions are evaluated and modified (Thompson 1994; Gross 1998). ER difficulties are linked with psychological disorders (Gross and Levenson 1997; Azad-Marzabadi and Amiri 2017). Research shows that poor and negative ER strategies are significant risk factors for the development and maintenance of psychological disorders (Betts et al. 2009; d'Acremont and Van der Linden 2007). Studies on ER and body image satisfaction, however, are scarce, such that greater use of avoidance strategies and less use of positive acceptance strategies were associated with greater body dissatisfaction. On the other hand, individual differences in reinforcement sensitivity have implications for the development of psychological disorders.

In reinforcement sensitivity theory (RST) (Gray 1970) differences in brain systems underlie individual personality and psychopathology (Deary 2009). In the original theory, the be- 
havioural activation system (BAS) is activated in response to reward, and the behavioural inhibition system (BIS) is activated in response to punishment (Deary 2009). In the substantial revisions of RST, the BIS has been divided into two system: the fight-flight-freeze system (FFFS) and the behavioural inhibition-anxiety system. RST suggests that the FFFS responds to aversive stimuli, whereas the revised behavioural inhibition-anxiety system resolves the approach versus avoidance of a stimulus and activation of the FFFS and BAS (Corr 2008).

According to revised reinforcement sensitivity theory (r-RST), impulsivity, anxiety, and fear are mediated via BAS, behavioural inhibitionanxiety, and FFFS, respectively (Bijttebier $e t$ al. 2009). Consistent with RST, high sensitivity of these systems may have an increased risk for psychological disorders (Bijttebier et al. 2009). Because elevated FFFS is a predisposing factor to phobia and panic, individuals with high levels of BAS activity are prone to addiction, and those with elevations of the BIS have an increased risk of anxiety disorders (Corr 2008). In addition to individual differences in reinforcement sensitivity, individuals process sensory information differently (Dunn 2001).

Some individuals may notice and respond to less intense or a greater number of stimuli in the environment than others. Individuals differ in how they neurologically transmit and process sensory information. Aron and Aron (1997) in their theoretical framework proposed that individuals with higher sensory-processing sensitivity (HSPS) are prone to perceive stimuli of lower intensity than others. These people, in situations where confronted with stimuli of higher intensity, are more easily overwhelmed and distressed.

The HSPS measures sensitivity to a variety of stimuli including pain, violent movies, caffeine, hunger, and loud noises. It also includes items that ask about feeling overwhelmed by intense sensory inputs. Other items include artistic or emotional sensitivity. Although research on HSPS is limited, some studies have shown that sensory sensitivity is associated with a variety of negative psychological outcomes, such as avoidant personality disorder (Meyer and Carver 2000), anxiety and depression (Liss et al. 2005), social phobia (Neal et al. 2002), and agoraphobic avoidance (Hofmann and Bitran 2007). Other studies provide evidence that the HSPS is related to stress (Benham 2006), and work-related variables (Evers et al. 2008).
The current study investigated the relationships between ER, reinforcement sensitivity and sensory-processing sensitivity, and attitudes towards appearance. It was hypothesised that there would be a positive association between FFFS and appearance, given that research has shown that the BIS has a reduced role in responses to punishment (Jackson 2009), and Gray and McNaughton (2000) conceptualise the BIS as a defensive approach system, while the FFFS is a separate defensive avoidance system. It was also hypothesised that negative and positive ER would be differently associated with body image dissatisfaction, although no research to date has investigated the relationship between r-RST (especially FFFS as a defensive avoidance system) and sensory sensitivity towards appearance. Finally, it is expected that ER plays an important role in sociocultural attitudes towards appearance.

\section{Material and methods}

\section{Participants and procedure}

The study participants were 331 students who were selected through multi-stage cluster sampling among students (public and private gifted schools) (Fig. 1). Inclusion criteria were being aged 18 years or less (minimum: 13 years, maximum: 18, and average: 15.3 ); based on self-report all participants reported that they had not been diagnosed with a medical condition (physical, but not necessarily psychological disorders) by a doctor. Participants with a psychotic disorder, problems with substance abuse, acute suicidality, insufficient language skills, or severe cognitive impairment were excluded.

Participants completed study questionnaires after informed consent was obtained. Of the total participants, 242 were in their first year of high school (73.1\%), 89 were in their second year

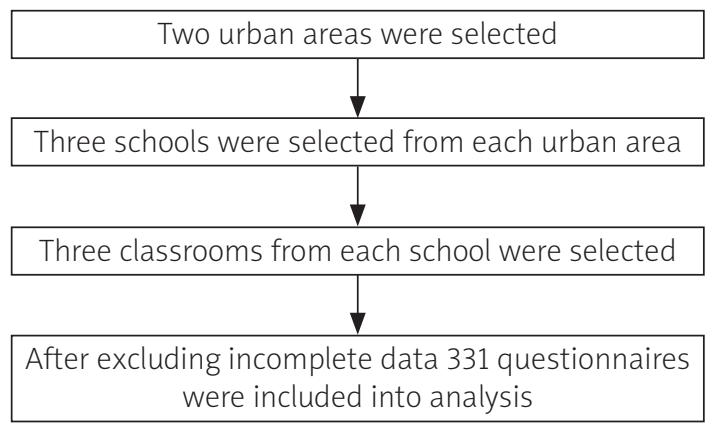

Fig. 1. Participant selection flowchart 
of high school (26.9\%), 195 participants were male $(58.9 \%)$, and 136 were female $(41.1 \%)$. Respectively, the mean age of the students and standard deviation were 14.25 and 0.87 in males, 14.21 and 0.95 in females.

\section{Measures}

\section{Sociocultural Attitudes Towards Appearance Questionnaire-4}

The Sociocultural Attitudes Towards Appearance Questionnaire-4 (SATAQ-4) is a 22-item questionnaire with five subscales: thin/low body fat, muscular/athletic, family pressures, peers pressures, and media pressures. This scale has good psychometric properties (Schaefer et al. 2014).

\section{Cognitive Emotion Regulation Questionnaire}

This questionnaire is an 18-item questionnaire measuring cognitive ER strategies (Garnefski and Kraaij 2006). There are nine subscales: selfblame, other-blame, rumination, catastrophising, putting into perspective, positive refocusing, positive reappraisal, acceptance, and refocus on planning.

\section{Reinforcement sensitivity}

This questionnaire is offered as an instrument to explore further the implications of approach and avoidance processes in personality. The final 21 items were shown to load on three factors: BIS, FFFS, and BAS, with seven items for each of the BIS, FFFS, and BAS factors (Cooper et al. 2016).

\section{Highly Sensitive Person Scale}

The Highly Sensitive Person Scale consists of 27 items that are rated from 1 (strongly disagree) to 7 (strongly agree). The items refer to three subscales: ease of excitation (EOE), aesthetic sensitivity (AES), and low sensory threshold (LST) (Aron and Aron 1997).

\section{Data analytic strategy}

SPSS version 22 (SPSS IBM, New York) was used to perform statistical analyses. Bivariate correlations were calculated to examine the associations among ER, brain behavioural systems, and sensory processing sensitivity with sociocultural attitudes towards appearance variables. Next, regressions were conducted to test the hypothesis that together ER, reinforcement sensitivity, and sensory processing sensitivity would provide significant incremental contribution, beyond each alone, in predicting each of the five dimensions of sociocultural attitudes towards appearance.

\section{Results}

Descriptive statistics and bivariate correlations among the study variables are presented in Table 1. Skew and kurtosis were within conventional range ( -1 to 1 ; George and Mallery 2003) for all of the variables.

Bivariate correlations showed that the five dimensions of sociocultural attitudes towards appearance (thin/low body fat, muscular/athletic, family, peers media pressures) were significantly positively associated with sensory processing sensitivity (see Table 1). The two dimensions of RST, including FFFS and BAS, were positively associated with some dimensions of sociocultural attitudes towards appearance, and BIS was positively associated with some dimensions of sociocultural attitudes towards appearance (see Table 1). The dimensions of ER included self-blame, rumination, positive refocusing, refocus on planning, putting into perspective, catastrophising, and other-blame were positively associated with some of the dimensions of sociocultural attitudes towards appearance, and some had a negative correlation (acceptance and positive reappraisal).

Multiple regression analyses were conducted to investigate the incremental validity of ER, reinforcement sensitivity, and sensory processing sensitivity domains when predicting the sociocultural attitudes towards appearance (see Table 2).

In the first set of regressions, ER domains were entered as a first step, followed by the reinforcement sensitivity and sensory processing sensitivity domains. RST domains explained 7\% of the variance and indicated FFFS and BAS as significant predictors; HSPS explained $4 \%$ of the variance and indicated ease of excitation as a significant predictor. After adding together ER, RST, and HSPS domains, the effects of FFFS and BAS were weaker and not significant, but the BIS effect became significant. For muscular/athletic, ER domains explained $16 \%$ of the variance, and this was driven by all ER domains except self-blame and acceptance. RST domains explained $9 \%$ of the variance and indicated FFFS and BAS as significant predictors; HSPS explained $18 \%$ of the variance and indicated ease of excitation and aesthetic sensitivity as significant predictors. After adding the ER, RST, and HSPS domains, only two ER domains 


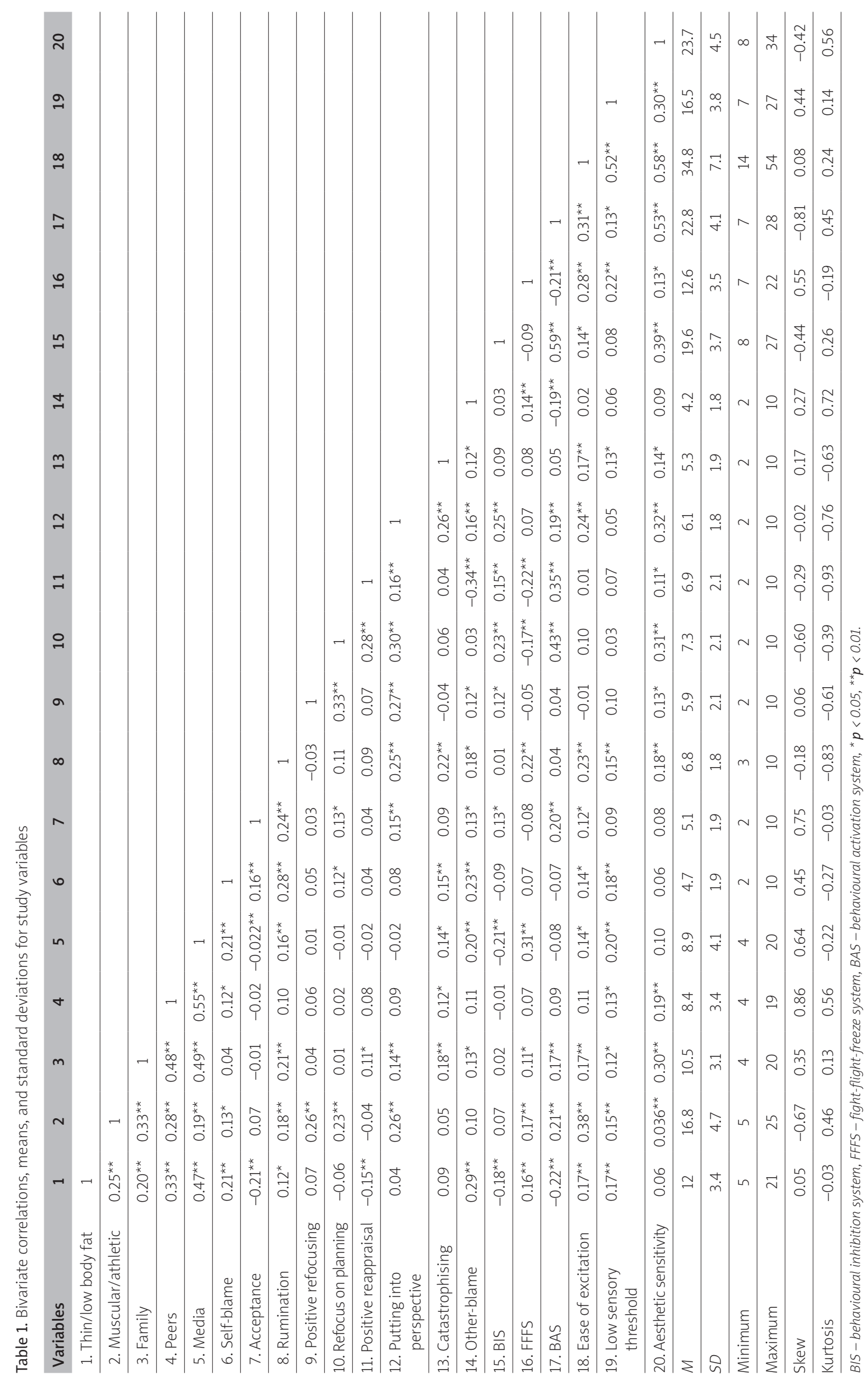




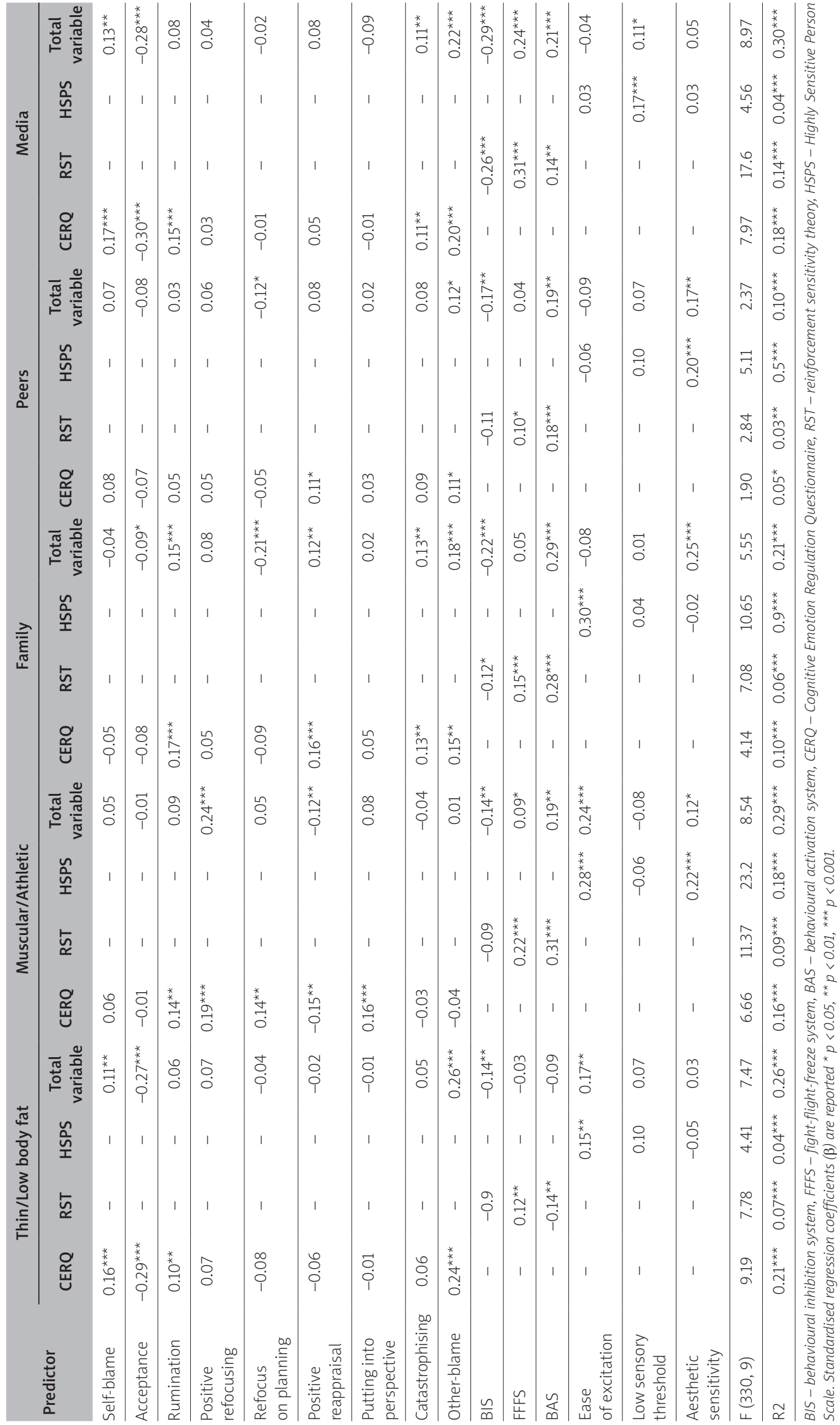


remained significant (positive refocusing and positive reappraisal), the effects of FFFS and BAS were weaker but remained significant, and the BIS effect became significant; the aesthetic sensitivity was weaker but remained significant. Finally, ER domains explained 6\%, 5\%, and $18 \%$ of the variance in family, peers, and media pressures, respectively, and this was mainly driven by negative ER domains (self-blame, rumination, catastrophising, and other-blame) and acceptance as positive ER domains (negative predictor). RST domains explained $6 \%, 3 \%$, and $14 \%$ of the variance in family, peers, and media pressures, respectively, and indicated FFFS and BAS predicted positively and BIS negatively. HSPS domains explained $9 \%, 5 \%$, and $4 \%$ of the variance, respectively, and indicated aesthetic sensitivity as a significant and stable predictor.

\section{Discussion}

As expected, sociocultural attitudes towards appearance were significantly positively related to negative ER (self-blame, rumination, and other-blame) in adolescents. The ways by which adolescents regulate their emotions were also significantly related to body image symptomatology. Attitude towards appearance was also more strongly related to greater use of internal dysfunctional ER strategies such as rumination. This suggests that general ER strategies may play an important role in relationships between body image concerns and other psychological symptoms.

These results indicate that adolescents who experience frequent negative thoughts and feelings about their appearance are more likely to have appearance dissatisfaction if they tend to use negative strategies to regulate negative emotions related to appearance, or if they tend to use internal dysfunctional ER strategies such as selfblame, rumination, and other-blame generally. Adolescents who experience frequent negative thoughts and feelings about their appearance tend to use internal dysfunctional ER strategies. These findings are consistent with past research reporting associations between dysfunctional ER and psychological symptoms (Betts et al. 2009).

Reinforcement sensitivity was significantly associated with greater thin/low body fat, muscular/athletic, family, and media pressures. These findings add knowledge to the revised RST and demonstrate the role of RST in psychopathology. This is the first study that has investigated the differential relationships of the revised RST to sociocultural attitudes towards appearance. Based on the revised RST, the FFFS was positively associated with appearance dissatisfaction, while the BIS was negatively associated with appearance dissatisfaction. Also, these findings add to the growing body of literature that investigates associations between high levels of reinforcement sensitivity and psychopathology. Hence, higher levels of FFFS sensitivity were associated with greater appearance dissatisfaction. Finally, the present study was one of the first attempts to explain the relationship between reinforcement sensitivity (e.g. FFFS sensitivity) and psychopathology.

One of the main goals of our study was to investigate the relationship between HSPS with body image aspects. Accordingly, the results supported the hypothesis that the three subscales of ease of excitation, low sensory threshold, and aesthetic sensitivity measured by the HSPS, according to Smolewska et al. (2006), exhibited a consistent pattern of associations with measure of sociocultural attitudes towards appearance.

The investigation of risk and protective factors related to body image dissatisfaction is an important avenue of research given the associations with pervasive disturbances. Although some notable attempts have recently been made to address sociocultural factors that may contribute to body image concerns (Australian Government 2010), the impact of such strategies is likely to be limited, and, moreover, it is improbable that all sources of body image concern (sociocultural) can be completely eradicated. Despite frequent attempts to develop interventions that promote positive body image, the success of such programs has thus far been disappointing, although some recent programs have shown promising results (Richardson and Paxton 2010; Littleton and Ollendick 2003). There is a clear imperative for research that advances the identification and understanding of factors that build resiliency against sources of body image concerns. Improving ER helps to reduce body image-related problems. In addition, ER training has been reported to reduce binge eating and depressive symptoms in individuals with eating disorders (Clyne and Blampied 2004).

There are several limitations of the current study, the first of which is its cross-sectional nature. Future studies that aim to assess for variables mediating the relationship between r-RST and attitudes towards appearance should ideally be prospective in nature and should assess for known comorbid disorders that are associated with body image disorder. 


\section{Conclusions}

In conclusion, the current study provides empirical support for the role of ER, r-RST, and HSPS in sociocultural attitudes towards appearance. This is the first known study to examine these relationships, and more studies are needed to better understand the underlying mechanisms.

\section{References}

1. Aron EN, Aron A. Sensory-processing sensitivity and its relation to introversion and emotionality. J Pers Soc Psychol 1997; 73: 345-368.

2. Australian Government. Voluntary industry code of conduct on body image. Retrieved from http://www.apo.org. au/research/voluntary-industrycode-conduct-body-image, 2010.

3. Azad-Marzabadi E, Amiri S. Morningness-eveningness and emotion regulation difficulty incremental validity in predicting social anxiety dimensions. Int I Gen Med 2017; 10: $275-279$

4. Benham G. The highly sensitive person: Stress and physical symptom reports. Pers Individ Dif 2006; 40: 1433-1440

5. Betts J, Gullone E, Allen JS. An examination of emotion regulation, temperament, and parenting style as potential predictors of adolescent depression risk status: A correlational study. Br J Dev Psychol 2009; 27: 473-485.

6. Bijttebier P, Beck I, Claes L, Vandereycken W. Gray's Reinforcement Sensitivity Theory as a framework for research on personality-psychopathology associations. Clin Psychol Rev 2009; 29: 421-430.

7. Cash TF, Fleming EC. The impact of body image experiences: development of the body image quality of life inventory. Int J Eat Disord 2002; 31: 455-460.

8. Cash TF, Phillips KA, Santos MT, Hrabosky JI. Measuring "negative body image": Validation of the Body Image Disturbance Questionnaire in a nonclinical population. Body Image 2004; 1: 363-372.

9. Cash TF. A "negative body image": Evaluating epidemiological evidence. In: Body image: A handbook of theory, research, and clinical practice, Cash TF, Pruzinsky T (eds.). The Guilford Press, New York 2002; 269-276.

10. Clyne C, Blampied NM. Training in emotion regulation as a treatment for binge eating: A preliminary study. Behav Change 2004; 21: 269-281.

11. Cooper AJ, Stirling S, Dawe S, et al. The reinforcement sensitivity theory of personality in children: A new questionnaire. Pers Individ Dif 2016; 115: 65-69.

12. Corr PJ. The Reinforcement Sensitivity Theory of Personality. Cambridge, UK: Cambridge University Press; 2008.

13. d'Acremont M, Van der Linden M. How is impulsivity related to depression in adolescence? Evidence from a French validation of the Cognitive Emotion Regulation Ouestionnaire. J Adolesc 2007; 30: 271-282.

14. Deary V. Punishment, Reward and Personality: A review of Corry, P.J. Pers Individ Dif 2009; 46: 565-566.

15. Dittmar H, Halliwell E, Banerjee R, et al. Consumer culture, identity and well-being: The search for the 'good life' and 'body perfect'. Psychology Press, New York 2007.

16. Dunn W. The sensations of everyday life: Empirical, theoretical, and pragmatic considerations. J Occup Ther 2001; 55: 608-620.

17. Evers A, Rasche J, Schabracq MJ. High sensory-processing sensitivity at work. Int J Stress Manag 2008; 15: 189-198.
18. Garnefski N, Kraaij V. Cognitive emotion regulation questionnaire - development of a short 18-item version (CERQ-short). Pers Individ Dif 2006; 41: 1045-1105.

19. George D, Mallery P. SPSS for Windows step by step: a simple guide and reference. 11. 0 update (4th ed.). Allyn \& Bacon, Boston, MA 2003.

20. Gray JA, McNaughton N. The neuropsychology of anxiety: An enquiry into the functions of the Septo-Hippocampal system. Oxford University Press, Oxford 2000.

21. Gray JA. The psychophysiological basis of introversion-extraversion. Behav Res Ther 1970; 8: 249-266.

22. Gross JJ, Levenson RW. Hiding feelings: The acute effects of inhibiting negative and positive emotion. J Abnorm Psychol 1997; 106: 95-103.

23. Gross JJ. Antecedent-and response-focused emotion regulation: Divergent consequences for experience, expression, and physiology. J Pers Soc Psychol 1998; 74: 224-237.

24. Hofmann SG, Bitran S. Sensory-processing sensitivity in social anxiety disorder: Relationship to harm avoidance and diagnostic subtypes. J Anxiety Disord 2007; 21: 944-954.

25. Jackson CJ. Jackson-5 scales of revised reinforcement sensitivity theory (rRST) and their application to dysfunctional real world outcomes. J Res Pers 2009; 43: 556-569.

26. Kostanski M, Gullone E. Adolescent body image dissatisfaction: Relationships with self-esteem, anxiety, and depression controlling for body mass. J Child Psychol Psychiatry 1998; 39: 255-262.

27. Liss M, Timmel L, Baxley K, Killingsworth P. Sensory processing sensitivity and its relation to parental bonding, anxiety, and depression. Pers Individ Dif 2005; 39: 1429-1439.

28. Littleton HL, Ollendick T. Negative body image and disordered eating behavior in children and adolescents: What places youth at risk and how can these problems be prevented? Clin Child Fam Psychol Rev 2003; 6: 51-66.

29. Meyer B, Carver CS. Negative childhood accounts, sensitivity, and pessimism: A study of avoidant personality disorder features in college students. J Pers Disord 2000; 14: 233-248.

30. Neal J, Edelmann RJ, Glachan M. Behavioral inhibition and symptoms of anxiety and depression: Is there a specific relationship with social phobia? Br J Clin Psycho 2002; 41: 361-374

31. O'Dea JA, Yager Z. Body image and eating disorders in male adolescents and young men. In P. I. Swain (Ed.), New developments in eating disorders research. Nova Science Publishers, Hauppauge, NY 2006; 1-36.

32. Richardson SM, Paxton SJ. An evaluation of a body image intervention based on risk factors for body dissatisfac tion: A controlled study with adolescent girls. Int J Eat Disord 2010; 43: 112-122.

33. Schaefer LM, Burke NL, Thompson JK, et al. Development and validation of the Sociocultural Attitudes Towards Appearance Questionnaire-4 (SATAQ-4). Psychol Assess 2014; 27: 54-67.

34. Smolewska KA, McCabe SB, Woody EZ. A psychometric evaluation of the Highly Sensitive People Scale: The components of sensory-processing sensitivity and their relation to the BIS/BAS and "Big Five". Pers Individ Dif 2006; 40: $1269-1279$

35. Thompson JK. Body image, eating disorders, and obesity in youth: The future is now. In: Body image, eating disorders, and obesity in youth: Assessment, prevention, and treatment, Thompson JK, Smolak L. American Psychological Association, Washington, DC; 2001; 1-21.

36. Thompson RA. Emotion regulation: A theme in search of definition. Monogr Soc Res Child Dev 1994; 59: 250-283. 